\title{
Modelling and quantitative analyses of Wolf-Rayet spectra: recent progress and results
}

\author{
Wolf-Rainer Hamann, Lars Koesterke, and Götz Gräfener \\ Universität Potsdam, Germany
}

\begin{abstract}
The quantitative analysis of Wolf-Rayet type spectra requires nonLTE models for expanding atmospheres which have been developed in the last decade. Idealizing assumptions of the so-called standard model are spherical symmetry, homogeneity and stationarity. While the first generations of models were restricted to simple atoms (helium, hydrogen), we now apply more advanced calculations which account for complex model atoms (nitrogen, carbon, oxygen). The re-analysis of the Galactic WN sample from the nitrogen lines led to a substantial revision of the stellar parameters, as far as the WNE-w subclass is concerned. Wind inhomogeneity ('clumping') has been accounted for in a first-order approximation. The degree of clumping is derived from the electron-scattering wings of strong lines. With clumped models, considerably lower mass-loss rates (by a factor two or more) are obtained, while other empirical parameters are not affected significantly. An overview is given about the fundamental parameters of Wolf-Rayet stars as obtained from spectral analyses, including new results on WN stars in the LMC.
\end{abstract}

\section{Introduction: the standard model for Wolf-Rayet atmospheres}

A 'standard' WR atmosphere is assumed to be expanding in a sphericallysymmetric, homogeneous and stationary flow. For the velocity field we adopt the usual $\beta$-law with $\beta=1$. The 'stellar radius' $R_{*}$, which is the inner boundary of our model atmosphere, corresponds per definition to a Rosseland optical depth of 20. The 'stellar temperature' $T_{*}$ is defined as the effective temperature referred to that radius $R_{*}$.

The radiation transfer is formulated in the comoving-frame of reference (CMF). Doppler broadening by 'microturbulence' is generally set to $100 \mathrm{~km} \mathrm{~s}^{-1}$. The radiation transfer is solved consistently with the set of statistical equations which account for all relevant radiative and collisional transition rates. The temperature stratification is calculated from the assumption of radiative equilibrium. Frequency redistribution of line photons by electron scattering is accounted by means of the appropriate angle-averaged redistribution function (Hummer 1962).

Any particular WR atmosphere is specified by its basic parameters $T_{*}, R_{*}$, $\dot{M}, v_{\infty}$ and chemical composition (e.g., given in the form of mass fractions $\beta_{\mathrm{He}}$, $\beta_{\mathrm{N}}$ etc.). Schmutz et al. (1989) discovered an interesting degeneracy in this parameter space. They defined a so-called transformed radius $R_{\mathrm{t}}$ as

$$
R_{\mathrm{t}}=R_{*}\left[\frac{v_{\infty}}{2500 \mathrm{~km} \mathrm{~s}^{-1}} / \frac{\dot{M}}{10^{-4} \mathrm{M}_{\odot} \mathrm{yr}^{-1}}\right]^{2 / 3}
$$


and found that models with same $R_{\mathrm{t}}$ exhibit almost the same emission line equivalent widths, irrespective of different combinations of $\dot{M}, R_{*}$ and $v_{\infty}$ (while, of course, $T_{*}$, composition etc. are fixed). This (approximate) invariance was validated by various numerical experiments with reasonable accuracy. In a stricter sense, one might compare only models with same terminal velocity $v_{\infty}$. Then even the line profiles and the total shape of the emergent spectra are invariant for models with same $R_{\mathrm{t}}$, except of a scaling of the absolute flux with $R_{*}^{2}$. This property greatly facilitates any spectral analyses, as the number of free parameters is reduced. Moreover, $R_{\mathrm{t}}$ has the advantage that it can be determined spectroscopically without knowing the distance of the object.

\section{WN analyses: from pure-helium to nitrogen models}

First generations of WR model atmospheres were restricted to pure-helium or helium-plus-hydrogen composition, due to the relative simplicity of these atoms. At the time of the last Wolf-Rayet symposium (IAU Symp. No. 163 in 1994), almost all existing spectral analyses thus were based only on these simple models.

Meanwhile, WN spectra have been analyzed extensively by means of their nitrogen lines. For the Galactic WN sample, the positions in the $\log T_{*}-\log R_{\mathrm{t}}$ plane are shown in Figure 1 (after Hamann \& Koesterke 1998a). The late subtypes (WNL) have stellar temperatures of $30-40 \mathrm{kK}$. Their parameters hardly changed, compared to the pure helium results, as indicated by the arrow for WR 156 as an example.

The early WN subtypes with strong lines (WNE-s) populate a strip from $T_{*}=45 \mathrm{kK}$ to $100 \mathrm{kK}$ and small $R_{\mathrm{t}}$, i.e., dense winds. Compared to the helium analyses, the nitrogen lines indicate slightly higher temperatures $(c f$. the shift vector for WR 136).

A bigger surprise came from the nitrogen analyses of the early WN subtypes with weak lines (WNE-w). From the pure-helium analyses, we had to adopt stellar temperatures below $40 \mathrm{kK}$ for that subclass in order to reproduce the observed He I lines. Now we obtain temperatures of $40-140 \mathrm{kK}$. This correction is substantial ( $c f$. the shift vector for WR 128 as example) and leads to considerably larger bolometric corrections, i.e., higher luminosities (see below). The hottest three WNE-w stars are those of earliest subtypes which could not be analyzed before with helium models because no He I lines are detectable in their spectra. Thus the WNE-w and WNE-s stars now cover the same temperature range, while the former have less dense winds (larger $R_{\mathrm{t}}$ ).

Why do we obtain those higher temperatures when analyzing WNE-w stars from their nitrogen spectra? The $\mathrm{N} v$ lines (which are present by definition in the early WN subtypes) require $T_{*} \geq 40 \mathrm{kK}$ in order to appear in the models. These $\mathrm{N} v$ lines are formed in deeper atmospheric layers. Therefore we think that they indicate the effective temperature more reliably than the He I lines which are formed in the outer parts of the atmosphere. The hotter (nitrogenfitting) models fail in reproducing the observed $\mathrm{He}$ I , but enhancing the density (mass-loss rate) by only a factor of two or so brings He I to the observed strength (while other lines then become much too strong). Part of this inconsistency is probably due to the neglection of line-blanketing in our models (line-blanketed 


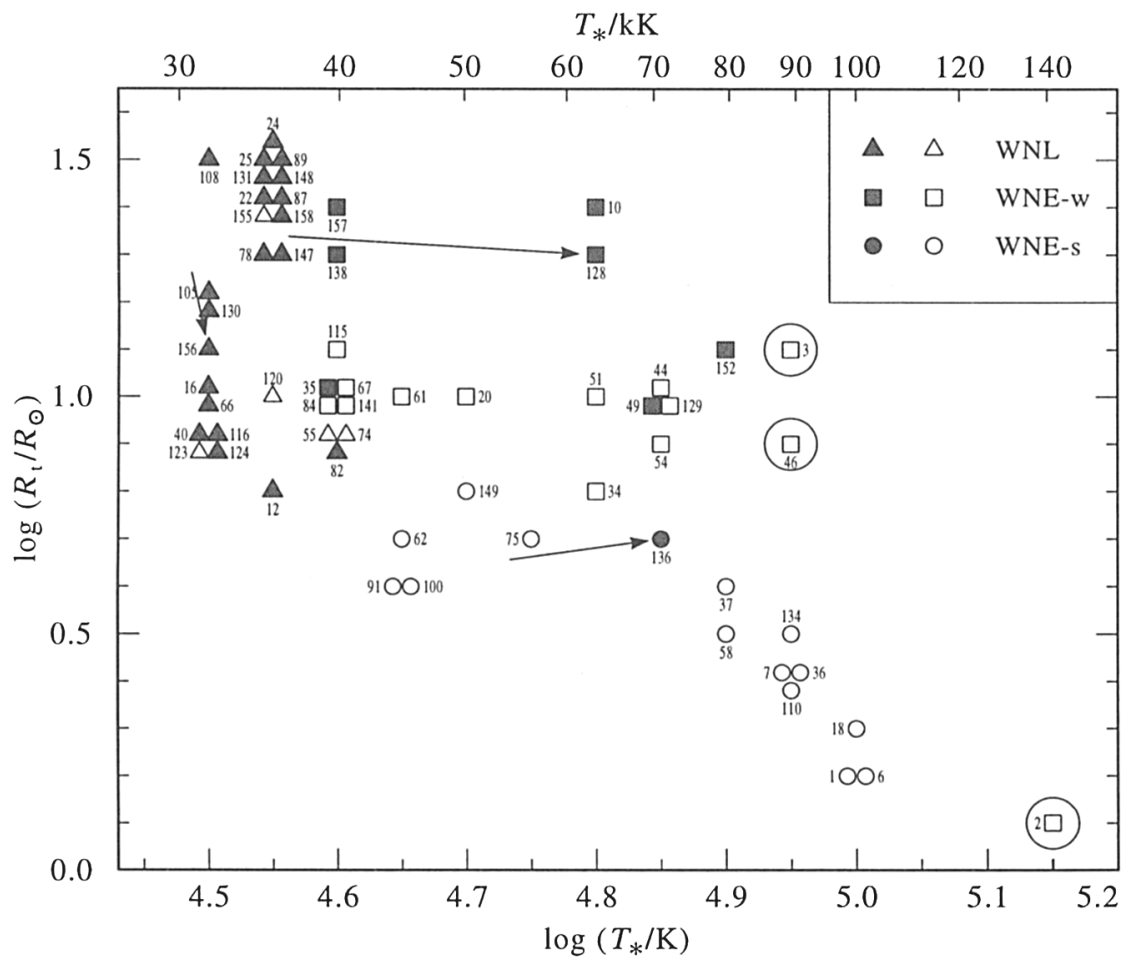

Figure 1. Positions of the Galactic WN stars (labels: WR catalogue numbers) in the $\log T_{*}-\log R_{\mathrm{t}}$-plane, as obtained from the analysis of the nitrogen spectra. The corrections against previous results from pure-helium analyses are indicated for one typical representative of each spectral subclass, showing that the temperature of the WNE-w stars has been significantly underestimated so far. Three stars of earliest subtypes (encircled) could be analyzed for the first time by using their nitrogen lines. Filled and open symbols distinguish between the presence and absence of atmospheric hydrogen, respectively.

models are just available now); furthermore, wind inhomogeneities might provide density enhancements which emit He I lines.

\section{The impact of clumping}

For a first-order approach to clumped stellar winds, we make the following simplifying assumptions which require only limited modifications in existing model codes ( $c f$. also Schmutz 1997):

- The wind consists of clumps with density $D \rho(r)$, where $\rho(r)$ is the density stratification of the homogeneous model with the same mass-loss rate. The factor $D>1$ thus gives the density enhancement, and is assumed to be constant all over the atmosphere.

- The interclump space is void. Thus the volume filling factor of the clumps is $f_{\mathrm{V}}=D^{-1}$. 
- The clumps are assumed to have small size, compared to the photon free path. Thus the radiative transfer can be calculated with 'effective' emissivities and opacities averaging between the clump and interclump medium.

Wolf-Rayet spectra are dominated by processes which scale with the square of the density. This can be concluded from the transformation invariance of Wolf-Rayet models described in the previous section ( $c f$. Hamann \& Koesterke $1998 \mathrm{~b}$ ). Therefore the concept of the 'transformed radius' can be generalized for clumped models. In order to cancel out in quadratic processes, the clump density enhancement $D$ must be compensated by diminishing the mass-loss rate by a factor $\sqrt{D}$. Thus we define

$$
R_{\mathrm{t}}=R_{*}\left[\frac{v_{\infty}}{2500 \mathrm{~km} \mathrm{~s}^{-1}} / \frac{\sqrt{D} \dot{M}}{10^{-4} \mathrm{M}_{\odot} \mathrm{yr}^{-1}}\right]^{2 / 3}
$$

and expect that models with same $R_{\mathrm{t}}$ exhibit similar spectra, irrespective of different combinations of $D, \dot{M}, R_{*}$ and $v_{\infty}$. Indeed, this degeneracy has been confirmed by numerical experiments (Figure 2; Hamann \& Koesterke 1998b).

Note that this scaling invariance holds only for the $\rho^{2}$ processes, i.e., for the main spectral features. Now, strong emission lines in Wolf-Rayet spectra exhibit extended line wings which are caused by line photons being scattered by free

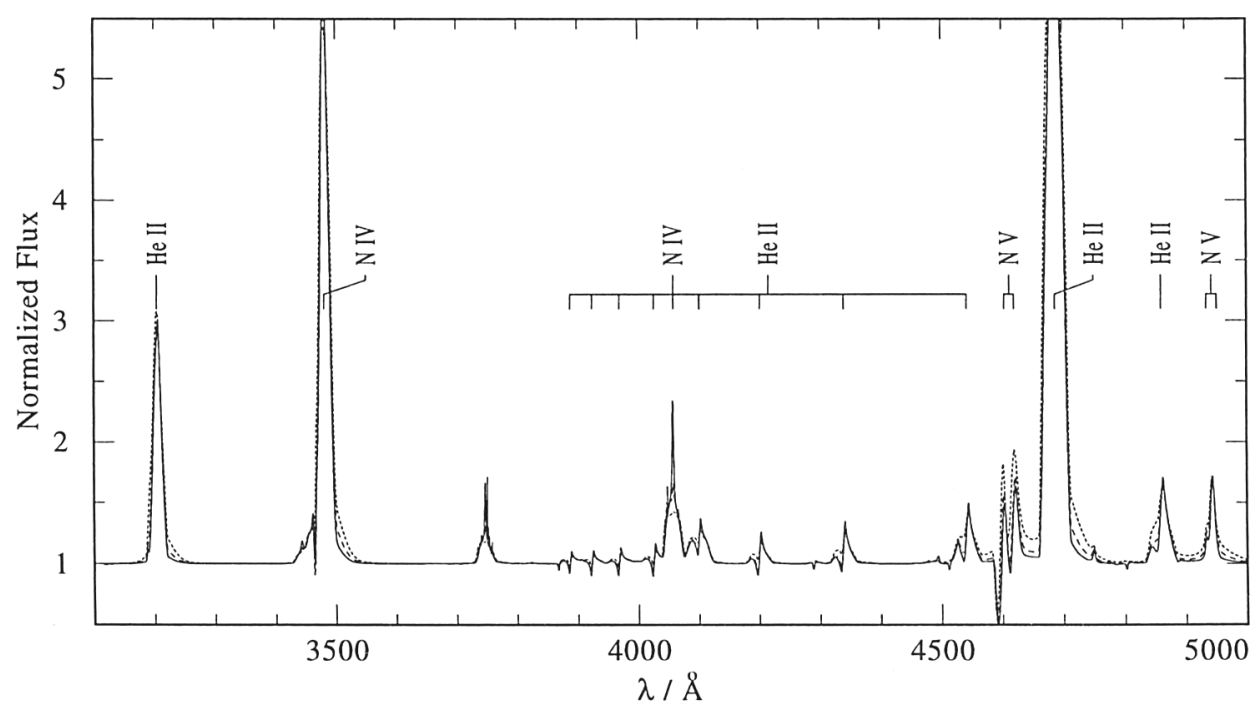

Figure 2. Numerical experiment, testing the validity of the scaling invariance with respect to clumping. We compare three models with different density contrast $D=1$ (short dashed), 4 (long dashed) and 16 (solid line). $R_{\mathrm{t}}$ is kept constant by a compensating change of the mass-loss rate $\left(\log \left(\dot{M} / \mathrm{M}_{\odot} \mathrm{yr}^{-1}\right)=-5.0,-5.3\right.$ and -5.6 , respectively $)$, while the other parameters are fixed $\left(\log L / \mathrm{L}_{\odot}=5.3, T_{*}=71 \mathrm{kK}, v_{\infty}=1600 \mathrm{~km} / \mathrm{s}, \beta_{\mathrm{He}}=0.985\right.$, $\left.\beta_{\mathrm{N}}=0.015\right)$. As expected, the strength of the emission lines remains almost constant along that series of models, while the electron-scattering wings become weaker with increasing clumping parameter $D$. 
electrons (Hillier 1984). The latter have a high thermal speed and thus efficiently redistribute the photons in frequency by Doppler shift. The electron scattering opacity, however, scales linearly with density. Thus, in inhomogeneous models the enhanced clump density is already fully compensated by the volume filling factor; scaling down the mass-loss rate in order to keep the same $R_{\mathrm{t}}$ decreases the effective Thomson opacity. Hence, for a series of models with same $R_{\mathrm{t}}$ the electron-scattering line wings become weaker with increasing $D$, while the main spectral feature remain unchanged. By fitting the observed electron-scattering line wings with model spectra we can determine the appropriate value of the density enhancement factor $D$.

From analyzing representatives of the different WN sublasses (Hamann \& Koesterke 1998b; Koesterke et al. these Proceedings) we obtained the best fits with $D=4$. In consequence of the described scaling invariance, thus all empirical mass-loss rates (as derived with homogeneous models) have to be scaled down

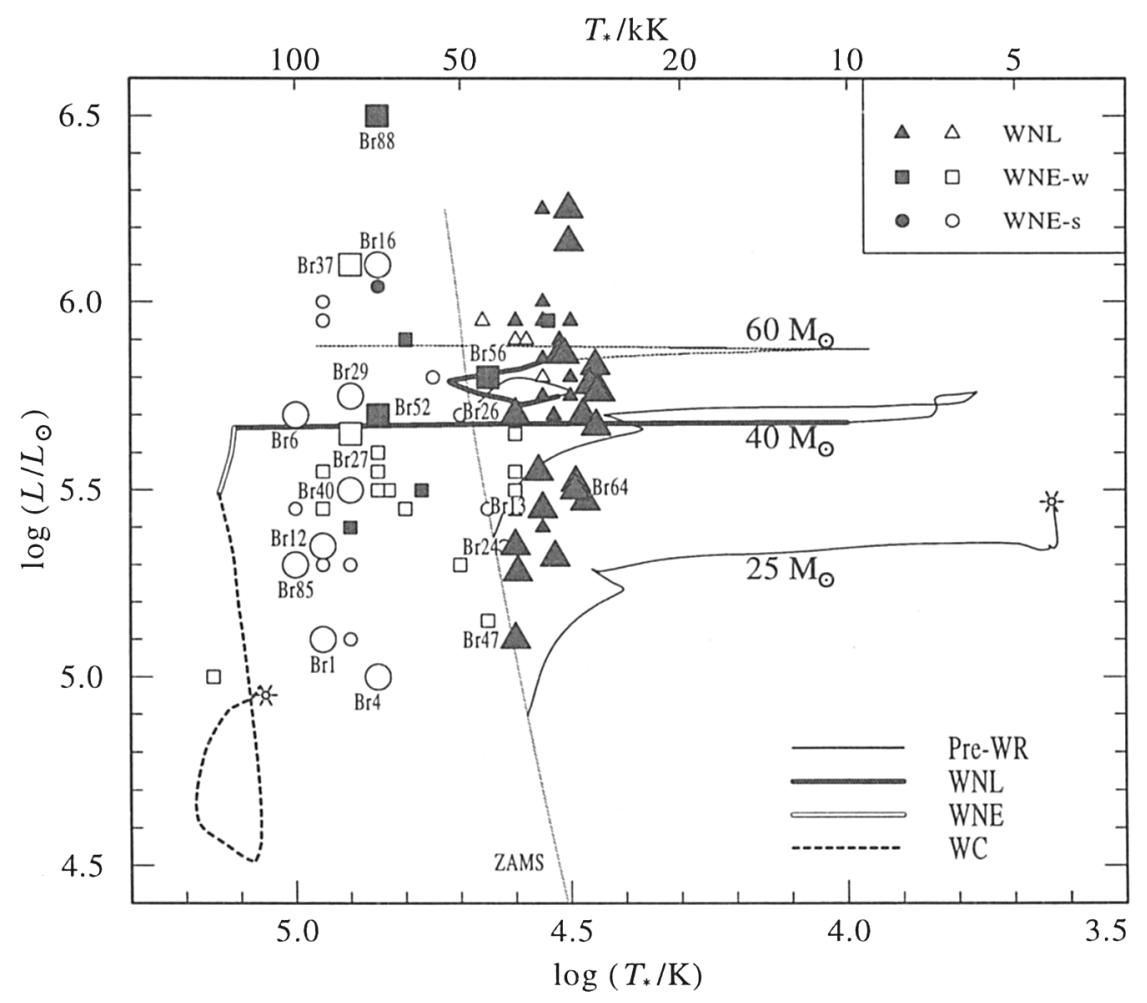

Figure 3. Analyzed WN stars in the HRD. Small, unlabeled symbols refer to Galactic objects. The big, labeled symbols denote recently analyzed LMC stars (Hamann \& Koesterke, in preparation), augmented by WNL stars (unlabeled big triangles) analyzed by Crowther \& Smith (1997). Filled and open symbols encode the presence and absence of atmospheric hydrogen, respectively. The Zero Age Main Sequence and theoretical tracks (25 and $40 \mathrm{M}_{\odot}$ : from Schaller et al. 1992 , set for $2 \times \dot{M}$; for $60 \mathrm{M}_{\odot}$ : alternative track from Langer et al. 1994) are shown just for better orientation. 
Table 1. Average ratio of mechanical to photon momentum, $\eta=\frac{\dot{M} v_{\infty}}{L / c}$

\begin{tabular}{lrrr}
\hline & \multicolumn{2}{c}{ Galaxy } & LMC \\
Subclass & $\eta$ (smooth) & $\eta$ (clumped) & $\eta$ (clumped) \\
\hline WNL & 9.1 & 4.5 & 6.0 \\
WNE-w & 8.7 & 4.3 & 1.7 \\
WNE-s & 29.4 & 14.7 & 17.9 \\
WC4 & & & 16.7 \\
\hline
\end{tabular}

by a factor of $\sqrt{D}=2$, while the other empirical parameters are not affected. WC stars have not yet been studied systematically, but our first impression is that their spectra fit best with even higher clumping, maybe $D \approx 16$.

The corresponding reduction of the empirical mass-loss rates has far-reaching consequences, e.g., for the calculation of evolutionary tracks or for the discussion of the wind driving mechanism. Table 1 shows the reduction of $\eta$, the ratio of mechanical wind momentum to the momentum of the radiation field, when clumping is taken into account. For the WN subclasses we adopt the

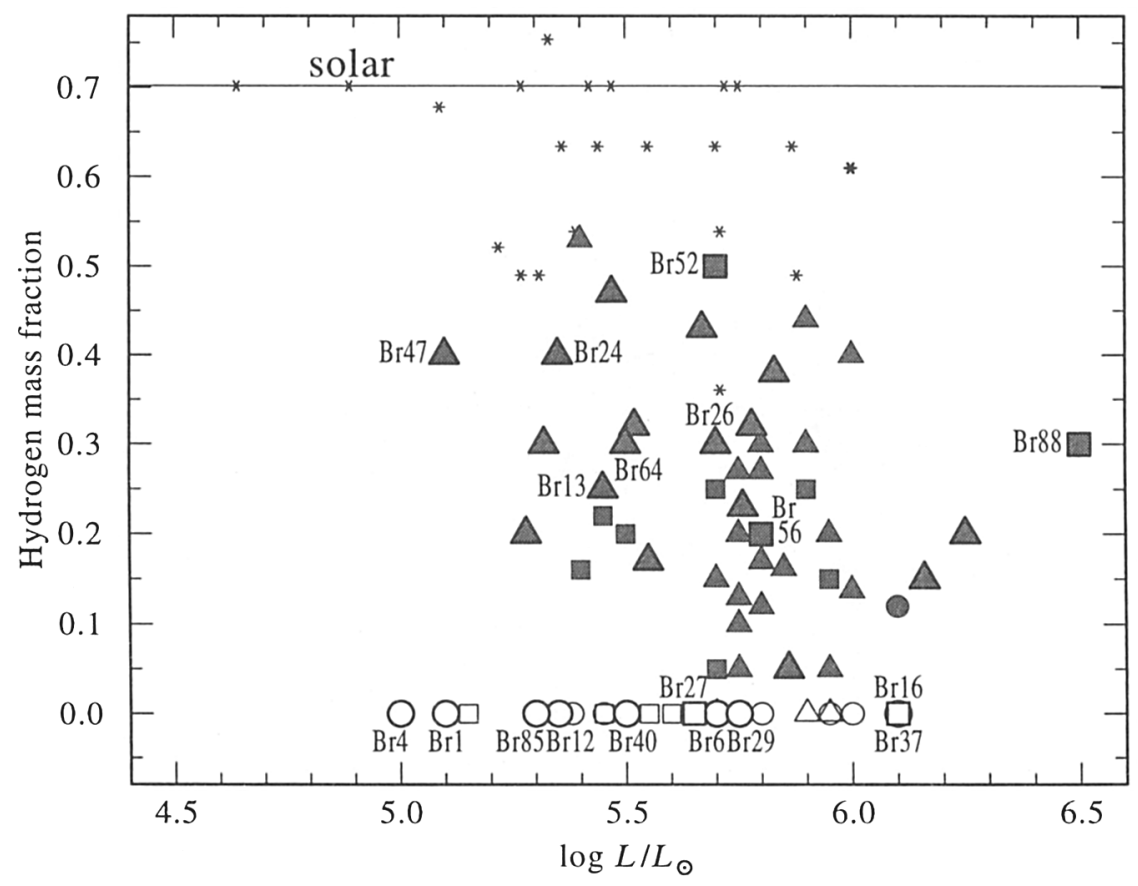

Figure 4. Hydrogen abundance versus luminosity for the analyzed WN stars. The smaller, unlabeled symbols refer to Galactic objects, while the bigger symbols stand for LMC stars (Br labels: Hamann \& Koesterke, in preparation; unlabeled: Crowther \& Smith 1997). Triangles, circles and squares indicate WNL, WNE-s or WNE-w subtypes, respectively. Small asterisks mark Galactic luminous OB stars (Herrero et al. 1992). 
average $\eta$ values from Hamann \& Koesterke (1998a) and $D=4$, while for WC stars the average for six WC4 stars (Gräfener et al. these Proceedings; Gräfener et al. 1998) is quoted and scaled according to $D=16 . \eta$ values of the order of ten are likely to be achievable by radiation driving, if enough line opacity is available (Lucy \& Abbott 1993) and the ionization structure is appropriate (Schmutz 1995).

\section{The fundamental parameters of the Wolf-Rayet stars}

The sample of known Galactic WN stars has been analyzed from the nitrogen spectrum (Hamann \& Koesterke 1998a; cf. Figure 1). The same technique has been applied recently to some $18 \mathrm{WN}$ stars in the LMC (Hamann \& Koesterke, in preparation), while Crowther \& Smith (1997) presented 20 analyses of LMC objects with WNL spectra. The distribution in the $\log T_{*}-\log R_{\mathrm{t}}-$ plane shows no difference between the Galactic and LMC members. The expected trend to weaker winds in the LMC (as consequence of smaller metallicity) is not visible in these data $c f$. also the average momentum ratios $\eta$ in Table 1). However, this

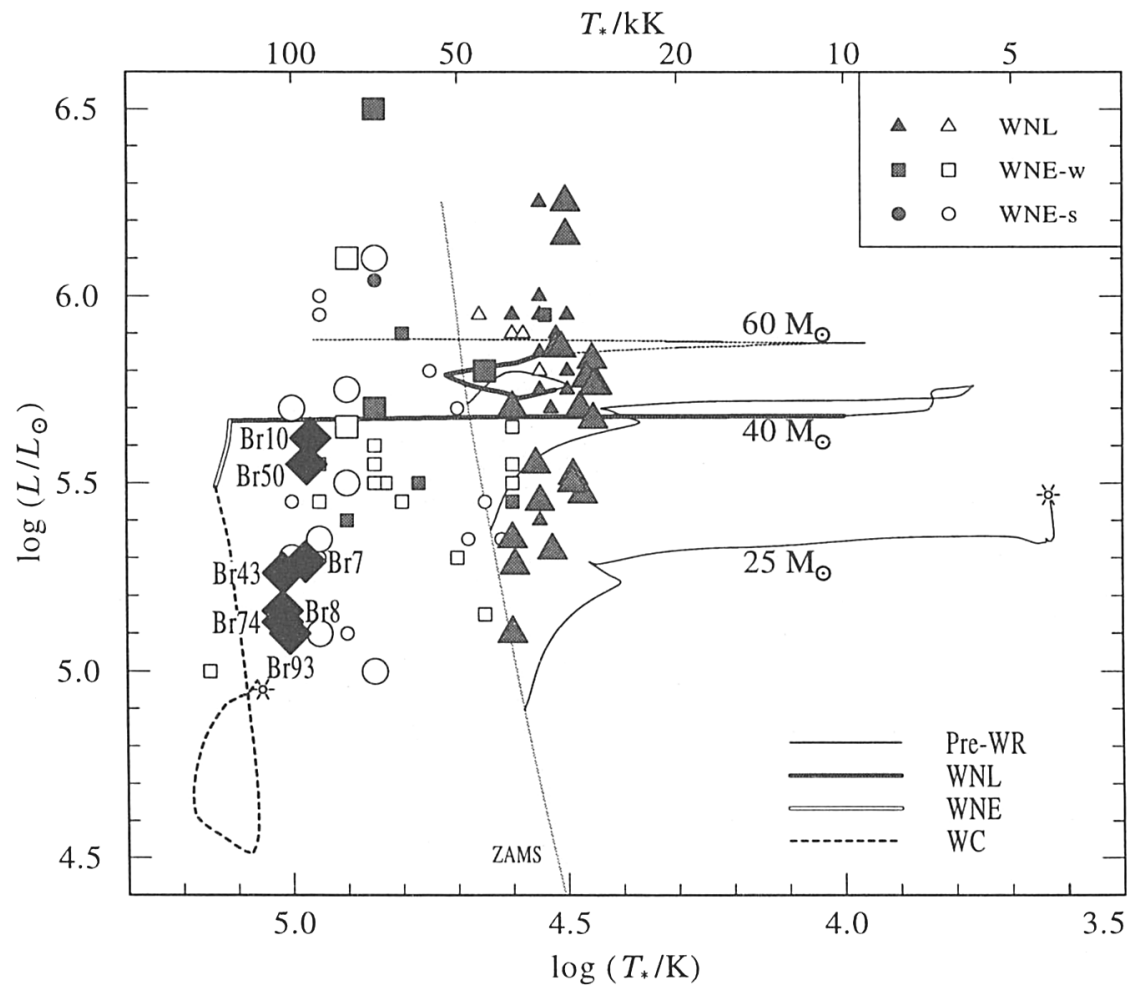

Figure 5. HR diagram (cf. Figure 3), additionally showing seven recently analyzed WC stars in the LMC (labeled symbols). Their spectral subtype is WC4, except Br 93 (WO4). 
comparison is biased by the different subtype composition of the Galactic and LMC subclass samples, respectively.

LMC stars have the decisive advantage that their distance is reliably known, while only a fraction of the Galactic WN stars can be attributed more or less definitely to specific open clusters or associations. Moreover, LMC stars are hardly reddened. Thus empirical luminosities of LMC stars are particularly accurate, the remaining source of uncertainty being only the bolometric correction taken from the fit model.

Figure 3 displays all analyzed WN stars in the HRD, emphasizing the LMC objects with big symbols. Luminosities range from $10^{5.0}$ to $10^{6.5} \mathrm{~L}_{\odot}$; the lower limit is not as low as obtained previously from pure-helium analyses, but still much lower than predicted by standard evolutionary tracks. The latter obviously do not reproduce the empirical HRD, probably due to neglect of rotationally induced mixing ( $c f$. Maeder, these Proceedings).

The importance of mixing processes is also evident from the atmospheric composition. Figure 4 shows that there is a continuously populated transition from initial surface composition to hydrogen-free atmospheres, while standard models predict a steep chemical profile.

WC stars have not been analyzed yet systematically, because of manifold difficulties. Recently, seven WC stars in the LMC have been analyzed by Gräfener et al. (these Proceedings; $c f$. also Gräfener et al. 1998), again taking advantage of the known distance to the LMC (Figure 5).

\section{References}

Crowther, P.A., Smith, L.J. 1997, A\&A 320, 500

Gräfener, G., Hamann, W.-R., Hillier, D.J., Koesterke, L. 1998, A\&A 329, 190

Hamann, W.-R., Koesterke, L. 1998a, A\&A 333, 251

Hamann, W.-R., Koesterke, L. 1998b, A\&A 335, 1003

Herrero, A., Kudritzki, R.P., Víilchez, J.M., Kunze, D., Butler, K., Haser, S. 1992, A\&A 261,209

Hillier, D.J. 1984, ApJ 280, 744

Hummer D.G. 1962, MNRAS 125, 21

Langer, N., Hamann, W.-R., Lennon, M., Najarro, F., Pauldrach, A.W.A., Puls, J. 1994, A\&A 290, 819

Lucy, L.B., Abbott, D.C. 1993, ApJ 405, 738

Schaller, G., Schaerer, D., Meynet, G., Maeder, A. 1992, A\&AS 96, 269

Schmutz W. 1995, in: K.A. van der Hucht \& P.M. Williams (eds.), Wolf-Rayet Stars: Binaries, Colliding Winds, Evolution Proc. IAU Symp. No.163 (Dordrecht: Kluwer), p. 127

Schmutz, W. 1997, A\&A 321, 268

Schmutz, W., Hamann, W.-R., Wessolowski, U. 1989, A\&A 210, 236

\section{Discussion}

Zhekov: (1) If we find the same density contrast over the entire wind, this may mean that the clumps are in pressure equilibrium with the smooth flow, so the temperature 
of the clumps will be lower than that of the smooth wind. (2) Do you find a different density contrast if you analize lines which are formed in different parts of the wind, e.g., at considerably different distances from the stellar surface?

Hamann: Electron-scattering wings are probing the clumping contrast close to the photosphere, where there is still some Thomson opacity. Radio mass-loss rates are in good agreement with mass-loss rates from emission lines, which tells us that clumping contrasts are not too different between the line-forming and the radio-emitting region (but see Nugis, Crowther \& Willis 1998, A\&A 333, 956). Temperatures are determined by radiative heating and cooling processes. Gas-pressure equilibrium is not a handle to conclude about temperatures.

Schaerer: None of the above talks have addressed the question of the He-, C-, Oabundances in WC and WO stars. Could you comment on this? Or is it premature to derive abundances for these stars from the current models?

Hamann: C- and O-abundances have been determined for six WC4 and one WO4 star in the LMC by Gräfener et al. (these Proceedings).

Maeder: You are suggesting a clumpiness factor of 4 for WN stars and about 16 for WC stars. Do you have an idea on the physical reason for this difference?

Hamann: I could think of two possible reasons: stronger instability of the radiationdriven wind, or stronger non-radial pulsations (if the latter are initializing the mass loss).

Georgiev: Did you find a dependence of the $\beta$ velocity-parameter with spectral subtype?

Hamann: Due to the high computational requirements, we have not yet studied different $\beta$ values systematically.

Crowther: The dependence of WR mass-loss properties on metallicity $Z$ is very important. Unfortunately, the Galaxy and the LMC are too close in $Z$ to make meaningful comparisons, as was already been demonstrated for O-type stars. Instead, we need to analyze individual WRs at lower $Z$ (which is problematic for the SMC because of the $100 \%$ binary WR frequency).

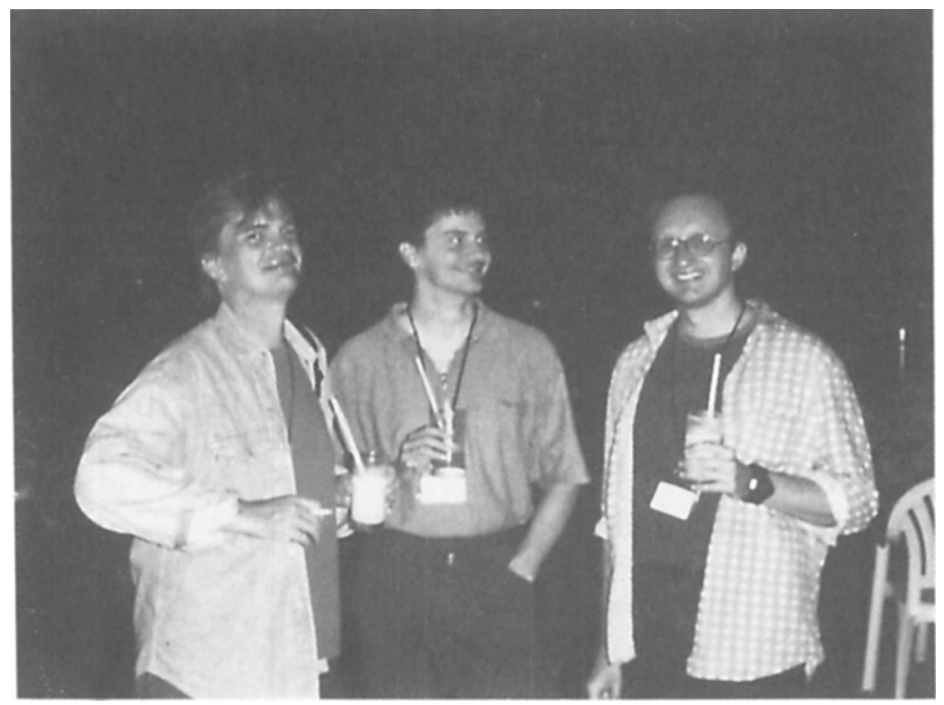

